\title{
Real-time images of tidal recruitment using lung ultrasound
}

\author{
Gerardo Tusman ${ }^{1 *}$, Cecilia M. Acosta ${ }^{1}$, Marco Nicola ${ }^{1}$, Mariano Esperatti ${ }^{2}$, Stephan H. Bohm³ \\ and Fernando Suarez-Sipmann ${ }^{4,5}$
}

\begin{abstract}
Background: Ventilator-induced lung injury is a form of mechanical damage leading to a pulmonary inflammatory response related to the use of mechanical ventilation enhanced by the presence of atelectasis. One proposed mechanism of this injury is the repetitive opening and closing of collapsed alveoli and small airways within these atelectatic areas - a phenomenon called tidal recruitment. The presence of tidal recruitment is difficult to detect, even with highresolution images of the lungs like $\mathrm{CT}$ scan. The purpose of this article is to give evidence of tidal recruitment by lung ultrasound.
\end{abstract}

Findings: A standard lung ultrasound inspection detected lung zones of atelectasis in mechanically ventilated patients. With a linear probe placed in the intercostal oblique position. We observed tidal recruitment within atelectasis as an improvement in aeration at the end of inspiration followed by the re-collapse at the end of expiration. This mechanism disappeared after the performance of a lung recruitment maneuver.

Conclusions: Lung ultrasound was helpful in detecting the presence of atelectasis and tidal recruitment and in confirming their resolution after a lung recruitment maneuver.

Keywords: Tidal recruitment, Atelectasis, VILI, Lung ultrasound, Recruitment maneuvers

\section{Background}

Tidal recruitment (TR) is the repetitive opening and closing of collapsed alveoli during the mechanical respiratory cycle $[1,2]$. The resultant high tissue stress in these atelectatic zones, and especially in the boundary areas between collapsed and open lung areas, can trigger a local inflammatory response and injure the alveolar-capillary membrane [3]. This is one of the proposed mechanisms of ventilator-induced lung injury (VILI) that impairs the outcome of patients with acute respiratory distress syndrome [1-4]. The other mechanism of VILI is tidal overinflation that could appear in some ventral areas of the lungs at the end of inspiration [5].

Nowadays, there is clear evidence that VILI can affect even patients with previous healthy lungs, the common scenario during general anesthesia [6-8]. This is

\footnotetext{
*Correspondence: gtusman@hotmail.com

${ }^{1}$ Department of Anesthesia, Hospital Privado de Comunidad, Córdoba

4545, 7600 Mar del Plata, Buenos Aires, Argentina

Full list of author information is available at the end of the article
}

of special relevance in surgical patients since there is an established link between atelectasis, VILI and the development of post-operative pulmonary complications [8-10].

The use of a lung protective ventilatory strategy resulted in a significant decrease in mortality in ARDS patients by the reduction of VILI during mechanical ventilation [4]. This ventilatory strategy minimized cyclic overdistensión and TR thereby decreasing the mechanical stress on lung tissue using low tidal volumes, plateau pressures and positive end-expiratory pressure (PEEP). However, this ventilation strategy does not fully eliminate TR because of the persistence of atelectasis along the mechanical ventilation period. Contrarily, lung recruitment-i.e. a ventilatory strategy that reverts atelectasis by applying a few mechanical breaths at high airways pressure-can potentially avoid the occurrence of VILI by TR $[8,11,12]$. This is simply because, by definition, TR is not possible without the presence of atelectasis.

Tidal recruitment is difficult to detect clinically and experimentally without the use of advanced imaging

\section{贷 Springer}


techniques. Currently, high-resolution CT scan is considered the gold standard method to detect TR in atelectatic areas by measuring the difference in aeration observed between static images taken at end-inspiration and endexpiration [12]. However, this method is expensive, time consuming; it submits the patient to radiation and cannot be applied at the bedside.

Lung ultrasound (LUS) is an attractive option to see TR in mechanical ventilated patients because, as opposed to $\mathrm{CT}$, it is non-invasive, radiation-free and simple to use at the bedside $[13,14]$. It has a high sensitivity and specificity for detecting lung collapse which appears as a LUS consolidation pattern $[13,15]$. The real-time nature of LUS images of atelectasis constitutes an important feature to detect this dynamic mechanism of VILI during the respiratory cycle. This short communication describes the use of LUS images to detect TR in mechanically ventilated patients during anesthesia and its resolution after applying a lung recruitment maneuver.

\section{LUS technique to assess TR}

LUS assessment was performed with the portable echograph MicroMax (Sonosite, Bothell, WA, USA) using a linear probe of 6-12 MHz. The echo probe placed in the longitudinal position along the ribs allowing for a systematic inspection of all lung areas [13, 15]. Once atelectasis areas were localized, two LUS's technical factors were crucial to detect TR in our patients. One factor was the use of a linear 6-12 MHz probe which provides highresolution images of atelectasis. The routine use of a linear probe is uncommon for lung studies in adult patients because it reaches a maximum depth of only $6 \mathrm{~cm}$. However, $6 \mathrm{~cm}$ of depth is enough to obtain a good view of the sub-pleural condensations with even more detail (figures and videos) $[15,16]$.

The other factor was to place the probe in the oblique position between the ribs to get a complete vision of the atelectatic area during the whole respiratory cycle [15]. Contrarily, applying the classical longitudinal approach (the "bat sign") could hide parts of atelectasis below the acoustic shadow of the ribs during the respiratory cycle, thus diminishing the view of the TR phenomenon.

\section{Clinical evidence of TR and its treatment by recruitment maneuver}

We present two clinical examples of how LUS easily detects TR and how lung recruitment maneuvers effectively eliminate such mechanism of VILI during anesthesia.

Figure 1 and Additional file 1: video S1 belong to a 68-year-old woman scheduled for surgical repair of multiple leg fractures. After induction of general anesthesia, protective ventilation was applied using volume-controlled ventilation $[4,6,10]$. This consisted of a tidal volume of

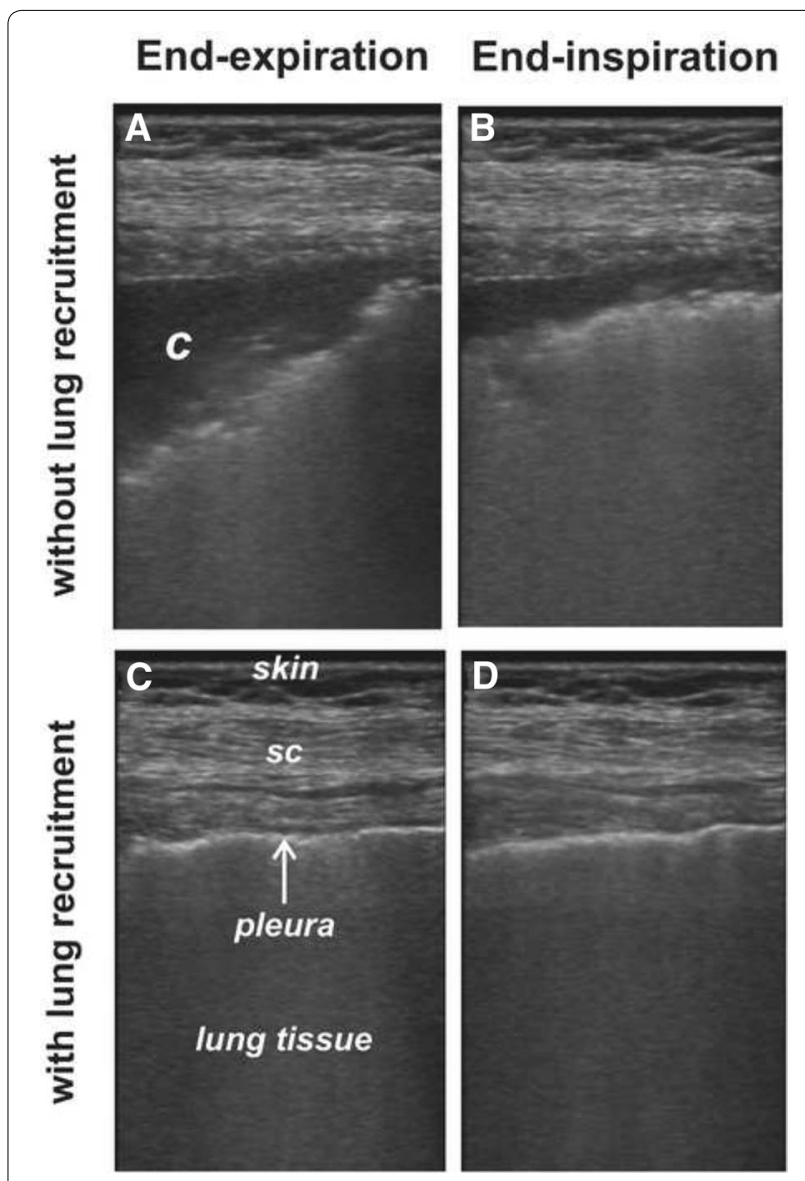

Fig. 1 Tidal recruitment in an anesthetized adult. All ultrasound images were obtained at the same lung region. Figures show atelectasis and tidal recruitment despite a protective ventilation pattern (A, B). A pulmonary consolidation (c) or atelectasis appears as a hypoechoic area mixed with airway bronchograms. Visual differences in the degree of lung aeration and the area of atelectasis between end-expiration and end-inspiration define the presence of tidal recruitment. $\mathbf{C}$ and $\mathbf{D}$ were obtained after a lung recruitment maneuver. They represent almost normal lung ultrasound images during end-expiration and end-inspiration, with the typical structures of skin, sub-cutaneous tissue (sc), pleural line and lung tissue. No differences in aeration were found during the breathing cycle

$6 \mathrm{~mL} / \mathrm{kg}$ ideal body weight, respiratory rate of $16 \mathrm{bpm}, \mathrm{I}: \mathrm{E}$ ratio of $1: 2$, PEEP of $8 \mathrm{cmH}_{2} \mathrm{O}$, and $\mathrm{FIO}_{2}$ of 0.6 . The corresponding plateau pressure was $27 \mathrm{cmH}_{2} \mathrm{O}$ and the finger pulse oximetry displayed a value of $94 \%$.

Ten minutes later LUS confirmed bilateral atelectasis in the most dependent lung areas, with the probe placed within the intercostal space at the posterior axillary line just above the diaphragm. This corresponds to the usual distribution of atelectasis during general anesthesia $[15,18]$. Most of the observed atelectatic area present at end-expiration (Fig. 1A) disappeared (i.e. was tidally recruited) at the end of the next inspiration (Fig. 1B; Additional file 1: video S1). 
We then performed a lung recruitment maneuver according to the method described by our group [19]. This ventilatory strategy consisted of a step-wise increment in airway pressure to reach the pressure that re-expands atelectasis. The response to lung recruitment was confirmed by the complete resolution of atelectasis observed in LUS images of the most dependent areas of the lungs $[15,17]$. Later on, a step-wise decrement in PEEP allowed the detection of the minimum level that prevents the reappearance of atelectasis in LUS images. After lung recruitment, ventilatory settings were returned to the protective ventilation pattern as described before, but this time maintaining the found PEEP level of $10 \mathrm{cmH}_{2} \mathrm{O}$ to maintain the lungs free of atelectasis. This ventilatory pattern resulted in a plateau pressure of $25 \mathrm{cmH}_{2} \mathrm{O}$ and a pulse oximetry reading of $99 \%$ using a $\mathrm{FIO}_{2}$ of 0.3 . LUS no longer showed neither atelectasis nor tidal recruitment (Fig. 1C, D; Additional file 1: video S1).

We observed similar findings in a healthy 3-year-old anesthetized child scheduled for laparoscopic surgery. Figure 2a, b shows atelectasis and TR in dependent, paradiaphragmatic lung areas using a protective ventilatory pattern. Figure $2 \mathrm{c}$, d obtained after a lung recruitment maneuver confirms the absence of atelectasis and tidal recruitment showing normal lung images. Additional file 2: video S2 presents the dynamic sequence of the corresponding images before and after lung recruitment.

\section{Commentary}

The role of LUS in detecting lung consolidation has been very well described and validated [13-15]. LUS is also useful to evaluate the effects of ventilatory strategies on atelectasis like PEEP or recruitment maneuvers [14, 15, $17,20,21]$. Such evaluation is based on the degree of lung aeration observed in the ultrasound images. However, to our knowledge, the diagnosis of TR by LUS has not been described before.

The presented cases illustrate how the atelectatic area appearing as a LUS consolidation pattern decreased during inspiration and returned to the original size at the end of expiration, confirming the presence of this mechanism of VILI in healthy lung ventilated with recommended lung protective settings during anesthesia. Furthermore, the elimination of the consolidated lung area by means of a lung recruitment maneuver confirmed the diagnosis of atelectasis and the consequent mechanism of TR.

\section{Conclusions}

Tidal recruitment is one of the principal described mechanisms producing VILI that affects mechanically ventilated patients with atelectasis. LUS can detect TR in an easy way by capturing its dynamic nature with real-time imaging. LUS also helped in confirming the

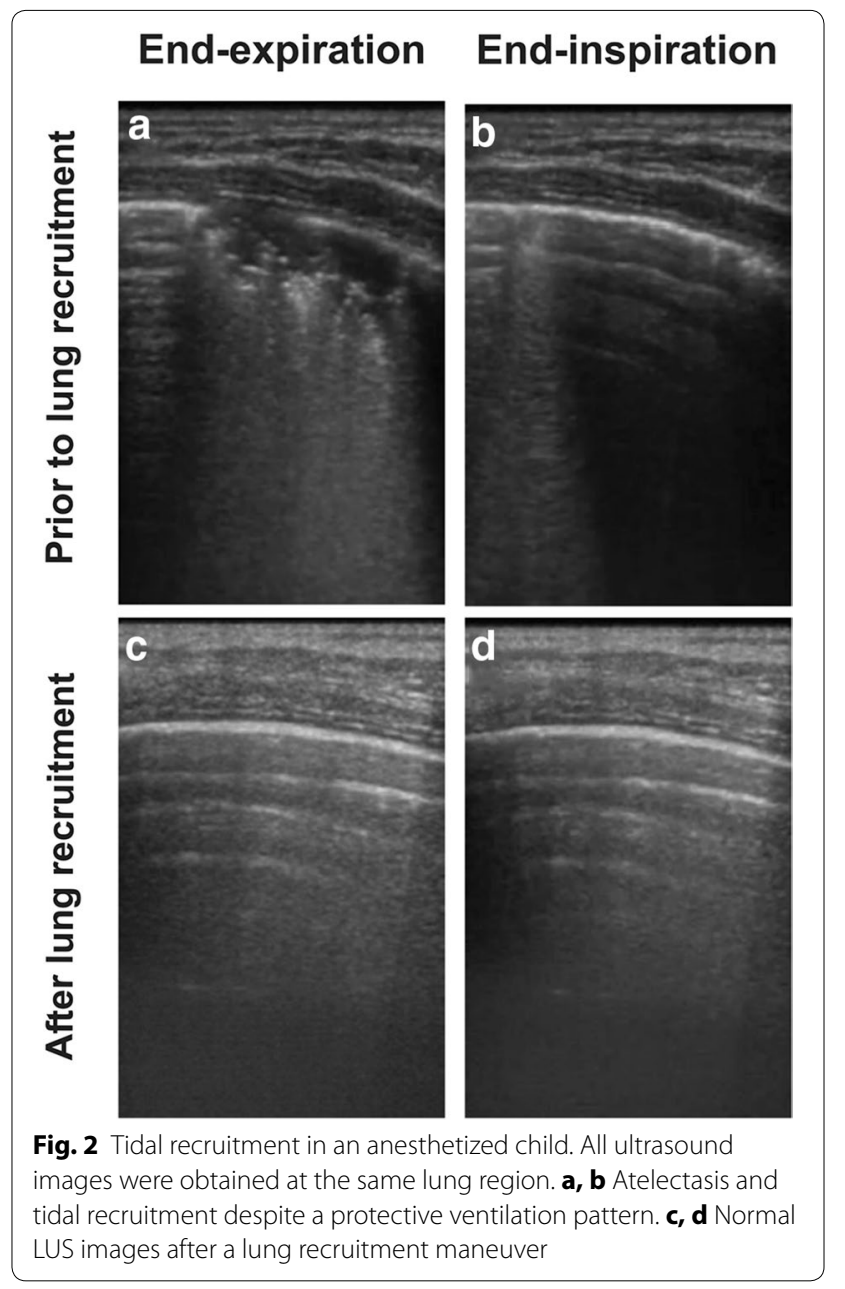

resolution of atelectasis and TR after lung recruitment and an individualized PEEP re-adjustment and can therefore become a valuable monitoring tool to prevent VILI at the bedside.

\section{Consent}

The institutional review board approved this publication and the corresponding written informed consent was obtained from the patient's relatives.

\section{Additional files}

Additional file 1: Video S1. This video shows the dynamic sequence corresponding to Fig. 1 in an anesthetized adult. Left: tidal recruitment can be seen during lung protective ventilation. Right: atelectasis and tidal recruitment disappeared after lung recruitment but using the same protective ventilatory pattern than before.

Additional file 2: Video S2. This video shows the dynamic sequence corresponding to Fig. 2 in an anesthetized child. Left: tidal recruitment before a lung recruitment maneuver. Right: images after lung recruitment. 


\section{Authors' contributions}

GT made the first case diagnosis and wrote the first draft. CMA and MN participated in the diagnosis and acquisition of data in the operating room. ME, FSS an SHB worked on the interpretation of the findings and in the final edition of the manuscript. All authors read and approved the final manuscript.

\section{Author details}

${ }^{1}$ Department of Anesthesia, Hospital Privado de Comunidad, Córdoba 4545, 7600 Mar del Plata, Buenos Aires, Argentina. ${ }^{2}$ Intensive Care Medicine, Hospital Privado de Comunidad, Mar del Plata, Buenos Aires, Argentina. ${ }^{3}$ Swisstom AG, Landquart, Switzerland. ${ }^{4}$ Department of Surgical Sciences, Section of Anesthesia and Critical Care Hedenstierna Laboratory, Uppsala University Hospital, Uppsala, Sweden. ${ }^{5}$ CIBER de Enfermedades Respiratorias, Instituto de Salud Carlos III, Madrid, Spain.

\section{Competing interests}

The authors declare that they have no competing interests.

Received: 11 September 2015 Accepted: 24 November 2015

Published online: 12 December 2015

\section{References}

1. Steinberg JM, Schiller HJ, Halter JM, Gatto LA, Lee HM, Pavone LA, Nieman GF (2004) Alveolar instability causes early ventilator-induced lung injury independent of neutrophils. Am J Respir Crit Care Med 169:57-63

2. Pavone LA, Albert S, Carney D, Gatto LA, Halter JM, Nieman GF (2007) Injurious mechanical ventilation in the normal lung causes a progressive pathologic change in dynamic alveolar mechanics. Crit Care 11:R64

3. Protti A, Andreis DT, Monti M, Santini A, Sparacino CC, Langer T, Votta E, Gatti S, Lombardi L, Leopardi O, Masson S, Cressoni M, Gattinoni L (2013) Lung stress and strain during mechanical ventilation: any difference between statics and dynamics? Crit Care Med 41:1046-1055

4. The National Heart, Lung, and blood Institute ARDS Clinical Trials Network. The Acute Respiratory Distress Syndrome Network (2000) Ventilation with lower tidal volumes as compared with traditional tidal volumes for acute lung injury and the acute respiratory distress syndrome. N Engl J Med 342:1301-1308

5. Terragni PP, Rosboch G, Tealdi A, Corno E, Menaldo E, Davini O, Gandini G, Herrmann P, Mascia L, Quintel M, Slutsky AS, Gattinoni L, Ranieri VM (2007) Tidal hyperinflation during low tidal volume ventilation in acute respiratory distress síndrome. Am J Respir Crit Care Med 175:160-166

6. Wolthius EK, Choi G, Dessing MC, Bresse P, Lutter R, Dzolic M, van de Poll T, Vroom MB, Hollmann M, Schultz MJ (2008) Mechanical ventilation with lower tidal volumes and positive end-expiratory pressure prevents pulmonary inflammation in patients without preexisting lung injury. Anesthesiology 108:46-54
7. Yang M, Ahn HJ, Kim K, Yi CA, Kim MJ, Kim HJ (2011) Does a protective ventilation strategy reduce the risk of pulmonary complications after lung cancer surgery? A randomized controlled trial. Chest 139:530-537

8. Futier E, Constantin JM, Paugam-Burtz C (2013) A trial of intraoperative low tidal volume ventilation in abdominal surgery. New Engl J Med 369:428-437

9. Tusman G, Böhm SH, Warner DO, Sprung J (2012) Atelectasis and perioperative pulmonary complications in high-risk patients. Curr Opin Anaesthesiol 25:1-10

10. Hemmes SNT, SerpaNeto A, Schultz MJ (2013) Intraoperative ventilator strategies to prevent postoperative pulmonary complications: a metaanalysis. Curr Opin Anesthesiol 26:126-133

11. Tusman G, Bohm SH, Suarez-Sipmann F (2013) Alveolar recruitment during mechanical ventilation. Where are we in 2013? Trends Anaesth Crit Care 3:238-245

12. Suarez-Sipmann F, Böhm S, Tusman G, Pesch T, Thamm O, Reismann $H$, Hedenstierna G (2007) Use of dynamic compliance for open lung positive end-expiratory pressure titration in an experimental study. Crit Care Med 35:214-221

13. Volpicelli G, Elbarbary M, Blaivas M, Lichtenstein DA, Mathis G, Kirkpatric AW (2012) Conference reports and expert panel: international evidencebased recommendations for point-of-care lung ultrasound. Intensive Care Med 38:577-591

14. Bouhemad B, Mongodi S, Via G, Rouquette I (2015) Ultrasound for "lung monitoring" of ventilated patients. Anesthesiology 122:437-447

15. Acosta C, Maidana GA, Jacoviti D, Belauzarán A, Cereceda S, Rae E, Ananda M, Gonorazky S, Bohm SH, Tusman G (2014) Accuracy of transthoracic lung ultrasound for diagnosing anesthesia induced atelectasis in children. Anesthesiology 120:1370-1379

16. Reissig A, Heyne JP, Kroegel C (2001) Sonography of lung and pleura in pulmonary embolism. Sonomorphologic characterization and comparison with spiral CT scanning. Chest 120:1977-1983

17. Bouhemad B, Brisson H, Le-Guen M, Arbelot C, Lu Q, Rouby JJ (2015) Bedside ultrasound assessment of positive end-expiratory pressure-induced lung recruitment. Am J Respir Crit Care Med 183:341-347

18. Brismar B, Hedenstierna $G$, Lundquist H, Strandberg A, Svensson L, Tokics $L$ (1985) Pulmonary densities during anestesia with muscular relaxation: a proposal of atelectasis. Anesthesiology 62:422-428

19. Tusman G, Bohm SH (2010) Prevention and reversal of lung collapse during the intra-operative period. Best Prac Res Clin Anaesthesiol 24:183-197

20. Arbelot C, Ferrari F, Bouhemad B, Rouby JJ (2008) Lung ultrasound in acute respiratory distress syndrome and acute lung injury. Curr Opin Crit Care 14:70-74

21. Gardelli G, Feletti F, Gamberini E, Bonarelli S, Nanni A, Mughetti M (2009) Using sonography to assess lung recruitment in patients with acute respiratory distress syndrome. Emerg Radiol 16:219-221

\section{Submit your manuscript to a SpringerOpen ${ }^{\circ}$ journal and benefit from:}

- Convenient online submission

- Rigorous peer review

- Immediate publication on acceptance

- Open access: articles freely available online

- High visibility within the field

- Retaining the copyright to your article

Submit your next manuscript at $>$ springeropen.com 